\title{
The effect of alpha asarone, olive oil, and dexamethasone on collagen-induced arthritis (CIA) in the mouse
}

\author{
Mayda Yanik Aquino-Vega ${ }^{1}$, Lorena Rodríguez-Páez ${ }^{2}$, Patricia Arce-Paredes ${ }^{1}$, \\ Víctor G. Hernández-Chávez ${ }^{3}$, Enrique Becerril-Villanueva ${ }^{1}$, Oscar Rojas-Espinosa $^{1}$ \\ ${ }^{1}$ Departamento de Inmunología, Escuela Nacional de Ciencias Biológicas, Instituto Politécnico Nacional, México D.F., México; \\ rojas_espinosa@hotmail.com \\ ${ }^{2}$ Departamento de Bioquímica, Escuela Nacional de Ciencias Biológicas, Instituto Politécnico Nacional, México D.F., México \\ ${ }^{3}$ Departamento de Morfología, Escuela Nacional de Ciencias Biológicas, Instituto Politécnico Nacional, México D.F., México
}

Received 13 December 2012; revised 16 January 2013; accepted 8 February 2013

\section{ABSTRACT}

Aim of the Study: The primary aim of the study was to test the effect of 2,4,5-trimethoxy-1-propenylbenzene (alpha asarone), a hypocholesterolaemic drug, on the progression of collageninduced arthritis (CIA) in mice. Olive oil, the vehicle of alpha-asarone, and dexamethasone were used as control treatments. Set-Up: Four groups of DBA/1 mice were immunised with chicken type II collagen (CII) via the intradermal route and either left untreated or were treated with alpha asarone, olive oil, or dexamethasone. A non-immunised group was an additional control. Follow-Up: The thicknesses of the rear and front footpads were continuously monitored, and the levels of anti-collagen antibodies were measured at the end of the experiment. The animals were then sacrificed, and their rear and front limbs were removed and processed for histological examination. Results: Alpha asarone had no anti-inflammatory effect on CIA, and in one-third of the animals, it showed a pro-inflammatory effect that was characterised by a marked accumulation of neutrophils. Olive oil did not show any obvious anti-inflammatory effect on $\mathrm{CIA}$, but it lowered the level of CII anti-bodies by $50 \%$, suggesting a potential long-term anti-inflammatory effect. As expected, dexamethasone had a clear anti-inflammatory effect on CIA. Conclusion: Alpha asarone did not show any antiinflammatory effect on CIA in the mice under the above conditions; however, the accumulation of neutrophils in the CIA lesions of mice treated with alpha asarone and the effect of olive oil in downregulating the levels of anti-CII antibodies in CIA are two findings that warrant further investigation.

Keywords: Collagen; Arthritis; Mouse; Asarone; Olive Oil; Dexamethasone

\section{INTRODUCTION}

"Elemuy" or "Yumel" (Guatteria gaumeri Greenman; Annonaceae), a native plant from Yucatan, Mexico, has been used in traditional medicine for the effective treatment of gallstones [1]. Because cholesterol is the major component of gallstones, the G. gaumeri preparation was suspected to have hypocholesterolaemic effects, and studies from Sánchez-Reséndiz et al. in rabbits supported this hypothesis [2]. The active ingredient in G. gaumeri was subsequently found to be 2,4,5-trimethoxy-1-propenylbenzene, or alpha asarone. This substance, which has been isolated from this and other plants by several methods [3,4] or has been synthesised [5-7], possesses the hypocholesterolaemic and cholelitholytic effects that were previously reported $[8,9]$. In hypercholesterolaemic rats, alpha asarone decreased serum LDL-cholesterol levels, stimulated bile flow, increased the secretion of bile salts, phospholipids and bile cholesterol and reduced the cholesterol levels in gallbladder bile, leading to a decrease in the cholesterol saturation index (CSI). These effects of alpha asarone satisfactorily explained the hypocholesterolaemic and cholelitholytic effects of the drug [10].

Olive oil is an integral ingredient of the Mediterranean diet, and it has proven effects in preventing the development of cardiovascular disease (CVD) [11] and colon and breast cancer, among other illnesses [12]. Olive oil has been found to counteract inflammation by significantly decreasing the levels of (soluble) intercellular ad- 
hesion molecule-1 (ICAM-1) and tumour necrosis factor alpha $(\mathrm{TNF} \alpha)$ [13]; vascular cell adhesion molecule (VCAM-1), ICAM-1, and TNF $\alpha$ have all been implicated in atherogenesis. Despite its anti-inflammatory qualities, the effect of olive oil in arthritis has not been widely tested but oleuropein aglycone, a major component of olive oil, ameliorated the clinical signs and histological changes in the joints and paws of mice with collageninduced arthritis (CIA) [14].

Dexamethasone is an anti-inflammatory drug that is largely used in the treatment of both experimental and clinical arthritis with satisfactory but sometimes variable results. For example, dexamethasone (DEX) repaired the articular surface and bone in zymosan-induced arthritis in the rat [15]; DEX significantly reduced the histologycal severity of synovitis and bone damage in mice that were intra-articularly injected with methylated bovine serum albumin (mBSA)[16], DEX protected against arthritis and joint destruction in ovariectomised mice with CIA [17]; compared with DEX alone, DEX phosphate encapsulated into liposomes markedly reduced the time that was required to treat adjuvant-induced arthritis in the mouse [18]; nine out of ten patients treated orally with DEX responded similarly to patients that were given DEX intramuscularly, and in both cases, a decrease in the disease activity score was observed [19]; and lastly, DEX lowered the levels of anti-citrullinated protein antibodies and rheumatoid factor in patients with arthralgia, although it did not prevent the development of arthritis [20].

In this work, we investigated the possible anti-inflammatory effects of alpha asarone and olive oil in comparison with dexamethasone in a mouse model of CIA. This model recapitulates the hallmarks of the human disease, including cellular infiltration, synovial hyperplasia, cartilage damage and bone erosion, all of which lead to joint pain and stiffness [21,22]. The CIA model has been widely used as an experimental model for the study of the immunopathology of arthritis and for the testing of anti-inflammatory drugs, including anticytokine antibodies [23-26].

\section{MATERIALS AND METHODS}

\subsection{Mice}

Adult $(20$ - $22 \mathrm{~g})$ female DBA-1(H-2 $\left.{ }^{\mathrm{q}}\right)$, BALB/c $\left(\mathrm{H}-2^{\mathrm{d}}\right)$, and $\mathrm{C} 57 \mathrm{BL} / 6\left(\mathrm{H}-2^{\mathrm{b}}\right)$ mice were purchased from Harlan (Mexico). Handling of the animals was performed according to the Mexican Official Norm (NOM062-ZOO-1999) for the Correct and Humane Handling of Experimental Animals.

\subsection{Chemicals}

Chemicals, including alpha asarone (2,4,5-trimeth- oxy-1-propenylbenzene), used in this study were purchased from Sigma Chemical Co. (Mexico), with the exception of complete Freund's adjuvant (4 mg BCG), which was purchased from MD Biosciences (Minnesota, USA).

\subsection{Isolation of Chicken Collagen Type II (CII)}

Collagen was isolated from the sternum of chickens following the procedure described by Inglis et al. [27] with minor modifications. Purified collagen was lyophilised and kept frozen $\left(-20^{\circ} \mathrm{C}\right)$ until use.

\subsection{Collagen Purity Assessment}

Purity of the collagen was assessed by electrophoresis in $10 \%$ polyacrylamide gels under reducing conditions in the presence of sodium dodecyl sulphate (PAGE-SDS$2 \mathrm{ME}$ ) according to the protocol described by Laemmli [28].

\subsection{Collagen-Induced Arthritis (CIA)}

Arthritis was induced by subcutaneously injecting 50 $\mu l$ of collagen type II, which is the major component of cartilage [29] in complete Freund's adjuvant (CFA) at each side of the tail base. The inoculum was prepared by admixing $6 \mathrm{mg}$ of collagen dissolved in $1.0 \mathrm{ml}$ of $0.1 \mathrm{M}$ acetic acid with an equal volume of CFA (4 mg BCG/ml). A boost was given 15 days later with the same amount of collagen in incomplete Freund's adjuvant.

\subsection{Arthritis Assessment}

Starting on day 20, daily measurements of the rear and front limb thicknesses were performed using a clock-type calliper (sensitivity of $0.01 \mathrm{~mm}$ ) (Mituyoyo 7301, Japan). Measurement was continued until day 65 - 67. The arthritis score was determined by averaging the thickness of the four (palm and pad) paws. Two measurements were taken from each paw.

\subsection{Corrective Treatment of Arthritis}

Groups of mice were either left untreated (control animals) or were inoculated with CII (CIA group). Twenty-one days after the primary immunisation, ten mice were treated with 1) alpha asarone (80 mg/kg); 2) extra pure olive oil $(0.1 \mathrm{ml})$; or 3$)$ dexamethasone $(0.18$ $\mathrm{mg} / \mathrm{kg}$ ). Treatment was administered on a daily basis through a gastric cannula (alpha asarone and olive oil) or by the intraperitoneal route (dexamethasone). The asarone dose was chosen based on previous experiments in which the dose induced a marked hypolipidemic activity in rats [10]. 


\subsection{Serum Sampling}

Mice were exsanguinated immediately after sacrifice, and the blood was allowed to clot to separate the serum. Sera were collected and stored frozen at $-20^{\circ} \mathrm{C}$ until use.

\subsection{Antibody Measurement}

Anti-collagen antibodies were measured in the sera of all experimental animals using an enzyme-linked immunosorbent assay (ELISA). The assay included 1) overnight coating of the ELISA wells with $2.0 \mu \mathrm{g}$ of CII in $0.1 \mathrm{ml}$ of $0.05 \mathrm{M}$ Tris- $0.2 \mathrm{M} \mathrm{NaCl}(\mathrm{pH} 7.5)$; 2) blocking of the wells with $0.2 \mathrm{ml}$ of $3 \%$ skimmed milk in $0.15 \mathrm{M}$ PBS for $30 \mathrm{~min}$; 3) incubation with $0.1 \mathrm{ml}$ of the sera diluted 1:1000 in skimmed milk for $2 \mathrm{~h}$; 4) incubation with $0.1 \mathrm{ml}$ of horseradish peroxidase (HRPO)-goat antimouse immunoglobulin antibody diluted 1:1000 in skimed milk for $1 \mathrm{~h}$; and 5) detection of the antigen-antibody complexes with $0.1 \mathrm{ml}$ of a chromogenic substrate consisting of $3 \mathrm{mg}$ of ortho-phenylenediamine and $10 \mu \mathrm{l}$ of $30 \%$ hydrogen peroxide in $10 \mathrm{ml}$ of $0.01 \mathrm{M}$ acetate buffer (pH 6.0). Wells were washed three times for $5 \mathrm{~min}$ each in PBS between steps. The absorbance of the coloured product was measured on an ELISA reader (Multiskan Plus, Labsystems, Finland) at $492 \mathrm{~nm}$.

\subsection{Lymphoproliferation Assays}

Splenocytes were prepared from the spleens of normal (control) mice and mice with collagen-induced arthritis. Cells at $5 \times 10^{5}$ per well in $0.2 \mathrm{ml}$ of DMEM-FCS were plated in quadruplicate into 96-well culture plates and stimulated with $10 \mu \mathrm{l}$ of either DMEM-FCS, concanavalin $\mathrm{A}(0.5 \mu \mathrm{g}$ per well), or CII (4.0 $\mu \mathrm{g}$ per well) for five days at $37^{\circ} \mathrm{C} / 4 \% \mathrm{CO}_{2}$. Twenty-four hours before the end of incubation period, $20 \mu \mathrm{l}$ of Alamar Blue (AbD Serotec, UK) was added to each well, and the plate was returned to the incubator. Emitted fluorescence was recorded using a Fluoroskan Ascent FL microplate reader (Thermo Electron Co., Finland) at $590 \mathrm{~nm}$.

\subsection{Histological Examination}

At the time of sacrifice (67 days after the primary immunisation with collagen), the front and rear limbs were removed, and the limb regions with hand and foot articulations were trimmed, fixed for $24 \mathrm{~h}$ in $4 \%$ paraformaldehyde in PBS, decalcified with 10\% EDTA (pH 6.0) for 6 weeks, and processed for paraffin sectioning using standard procedures. Four-micron-thick sections were prepared in a Leica RM2125 (Biosystems Nussloch $\mathrm{GmbH})$ microtome, and the sections were stained with haematoxylin-eosin for general histology and with Masson's stain for collagen fibres [30]. Microphotographs were taken with a Nikon E800 C-LP microscope (Japan) and edited with Jasc Paint Shop Pro 9 software.

\subsection{Neutrophils}

Neutrophils were identified in paw sections using rat antibodies to the mouse neutrophil markers, GR-1 and 7/4. After boiling in antigen retrieval solution (Dakocytomation, Carpinteria, CA, USA) for $30 \mathrm{~min}$, slides were washed with tap water and PBS. Non-specific binding was prevented by adding 5\% normal donkey serum and Fc block $(5 \mu \mathrm{g} / \mathrm{ml}, 2.4 \mathrm{G} 27$, Trudeau Institute Antibody Core Facility) for $30 \mathrm{~min}$, and the slides were then incubated overnight at room temperature with rat biotinylated antibodies to 7/4 (AbD Serotec, Raleigh, NC, USA) and rat anti-mouse Ly-6C and Ly-6G (Gr-1, BD Pharmingen, clone RB6-8C5; San Diego, CA, USA). Neutrophils $\left(7 / 4^{+} \mathrm{Gr}-1^{+}\right)$were visualised by adding a donkey anti-rat antibody coupled to Alexa Fluor $568(610 / 570 \mathrm{~nm})$ and streptavidin-Alexa Fluor 555 (580/550 nm) (Molecular Probes, Eugene, OR, USA). After washing in PBS, the slides were mounted with Fade Prolong Gold AntifadeDAPI (Molecular Probes, Eugene, OR, USA).

\subsection{Statistical Analysis}

All experimental groups were analysed in comparison to the control group by the Kruskal-Wallis one way analysis of variance on ranks and the Dunn's method for multiple comparisons.

\section{RESULTS}

\subsection{Collagen-Induced Arthritis (CIA)}

Neither BALB/c $\left(\mathrm{H}-2^{\mathrm{d}}\right)$ nor C57BL/6 $\left(\mathrm{H}-2^{\mathrm{b}}\right)$ mice developed arthritis following the immunisation protocol described in the materials and methods section. By contrast, most DBA/1 mice $\left(\mathrm{H}-2^{\mathrm{q}}\right)(90 \%$ - 100\%) developed arthritis in both the rear and front footpads (Figure 1). In the absence of treatment, arthritis began to be evident by day 21 after the primary immunisation and remained until the end of the study on day 67 (Figure 2).

\subsection{Lymphoproliferation}

As expected, the mice that developed CIA were characterised by the presence of CII-activated lymphocytes. Normal mice underwent lymphoproliferation in response to concanavalin A (Con-A), but they were non-responders to CII, whereas CIA mice responded both to Con-A and CII. Interestingly, the splenocytes of CIA mice showed a high degree of activation in the absence of any stimulus, and this proliferative response was not surpassed by the response induced with Con-A or CII (Figure 3). 

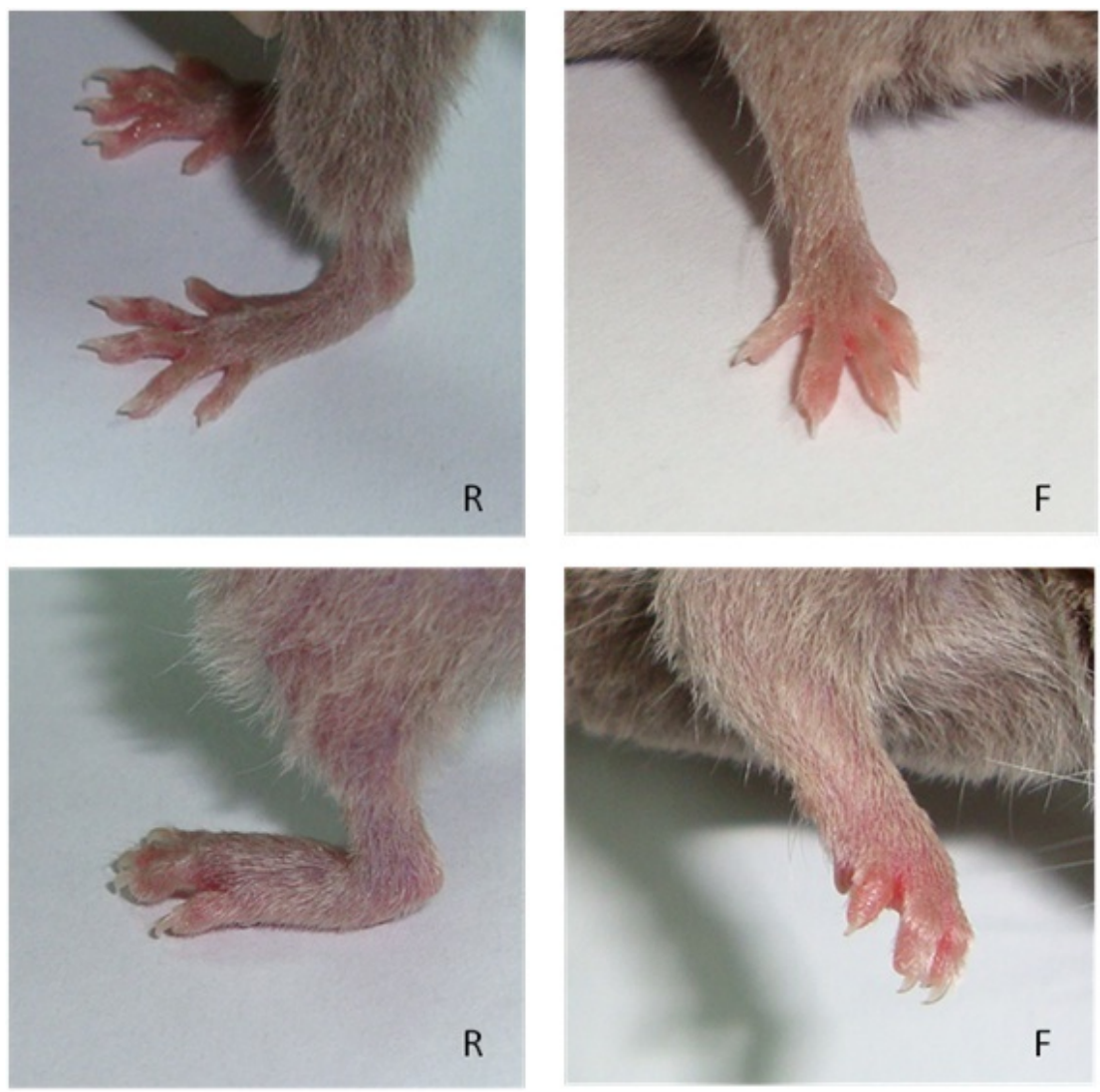

Figure 1. Appearance of the rear $(\mathrm{R})$ and front $(\mathrm{F})$ footpads of a normal mouse (upper pair) and the rear and front footpads of a mouse with collagen-induced arthritis (CIA) (lower pair) 40 days after primary immunisation.

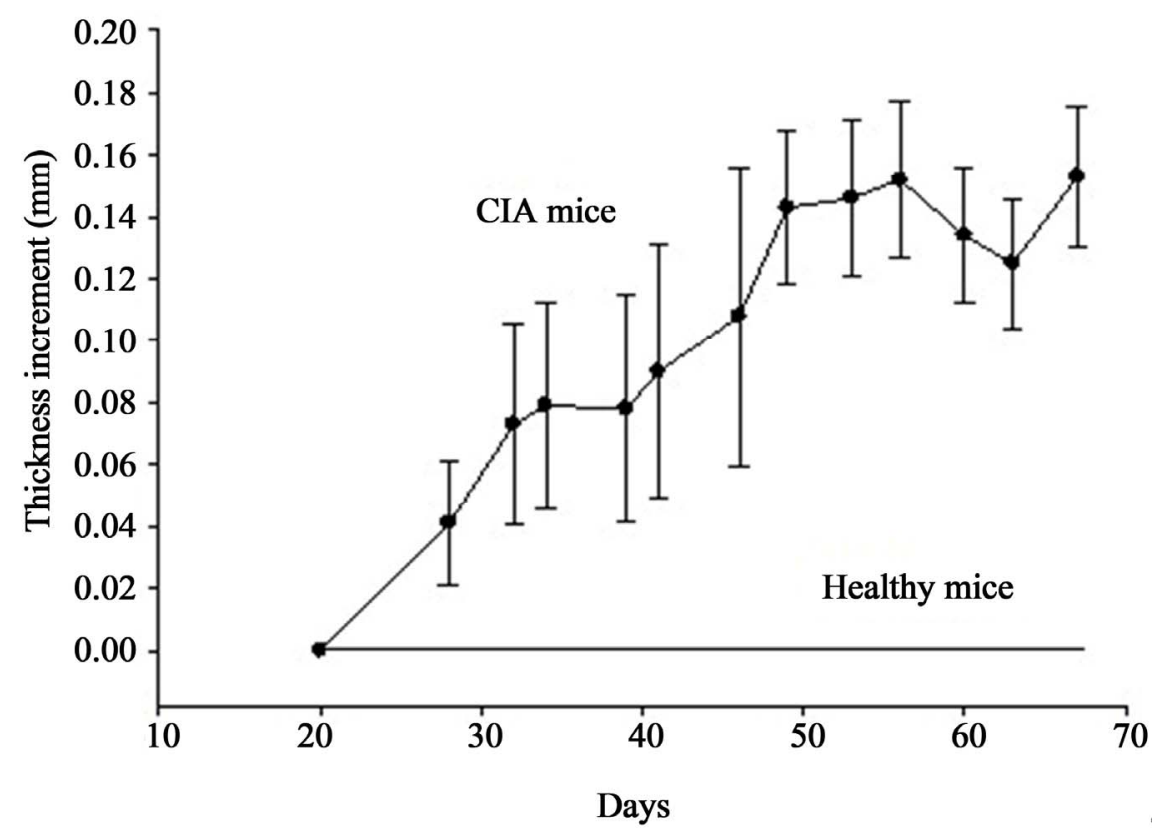

Figure 2. Evolution of CIA in DBA/1 mice. Because of the intrinsic variation in each mouse, data were corrected by subtracting the average thickness of the normal rear footpads from the thickness of the rear footpads of CIA mice measured on the same day (10 mice per group). 


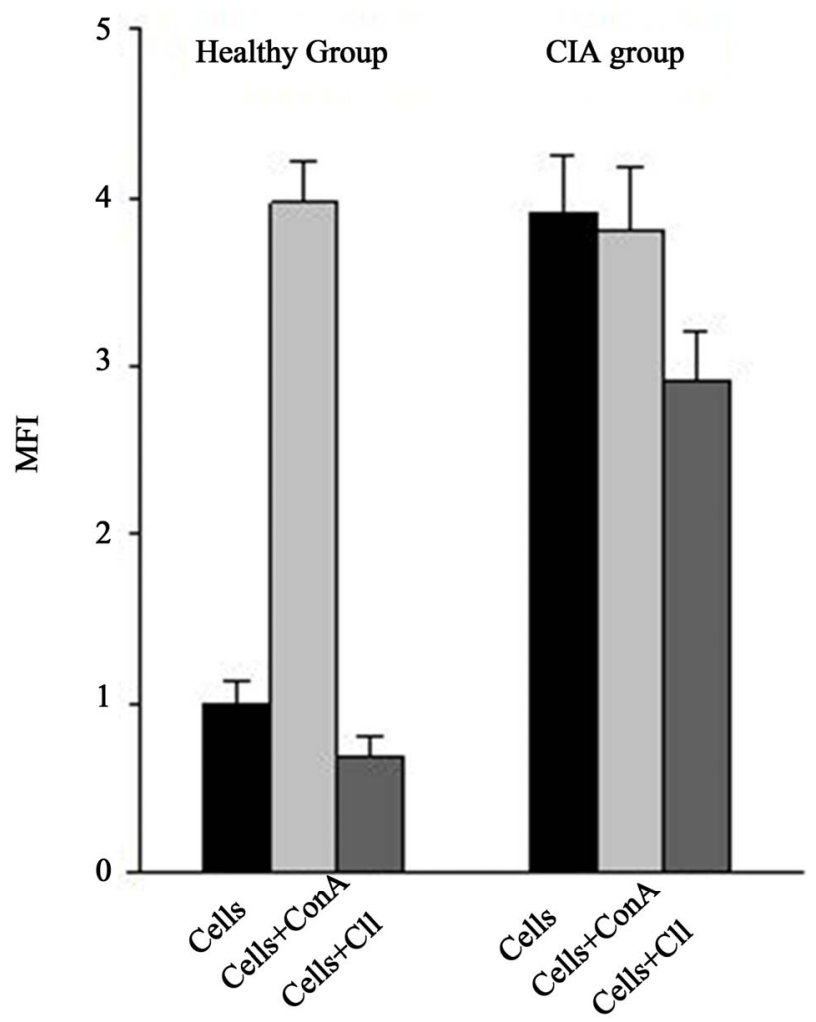

Figure 3. Proliferation of spleen cells from healthy and CIA mice in response to ConA or CII. Mean Fluorescence Index (MFI) values were normalised to the MFI values in healthy, non-stimulated mice $(\mathrm{P}<0.001)$.

\subsection{Effect of Alpha Asarone on CIA}

Compared with the mice with CIA, no anti-inflammatory response was observed in the arthritic mice that were treated with alpha asarone (Figure 4). Furthermore, some alpha asarone-treated mice showed a higher degree of inflammation as evidenced in the histopathological analysis of the footpads.

\subsection{Effect of Olive Oil on CIA}

Mice treated with olive oil alone showed a comparable degree of inflammation to the animals immunised with collagen (Figure 4).

\subsection{Effect of Dexamethasone on CIA}

Dexamethasone had an anti-inflammatory effect on CIA that began to be evident approximately 8 days after onset of CIA (Figure 4).

\subsection{Histological Findings}

The following descriptions take into account the overall changes that were observed within each experimental group, and representative images are shown for each group.

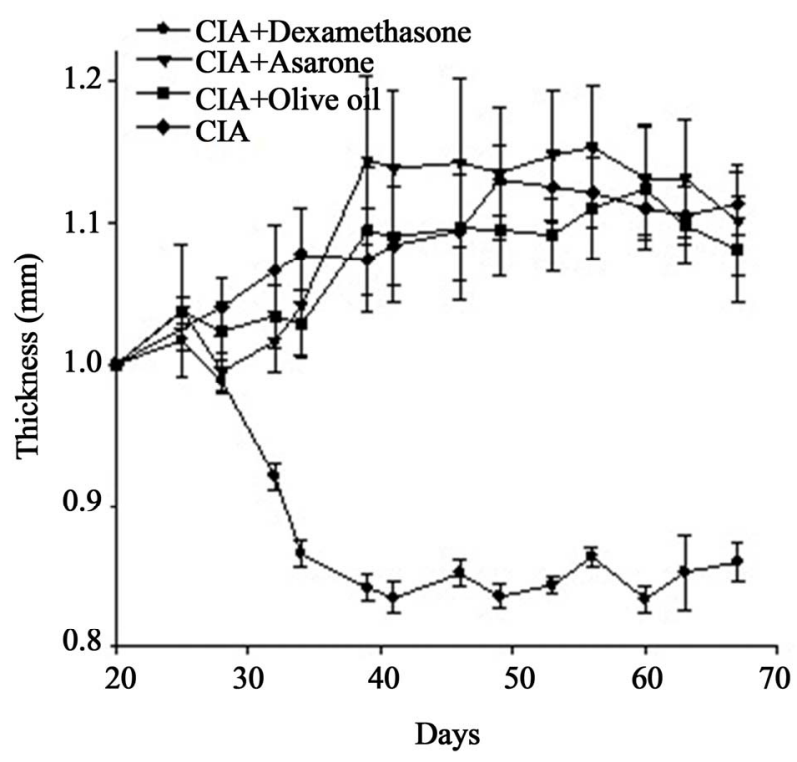

Figure 4. Effect of dexamethasone, alpha asarone, or olive oil on collagen-induced arthritis in DBA/1 mice. Values were normalised to the first thickness measurement in the CIA group.

\subsection{Healthy Mice (H)}

Sections from this group show skin, annexes and soft tissue, and none of these tissues exhibited noticeable alterations. The vascular and nerve elements also appeared normal. The haematopoietic tissue showed no anomalies, and the articular structures indicated that the integrity of the subchondral bone and cartilage was maintained and that no proliferative or inflammatory processes of the bursa or synovia occurred (Figure 5).

\subsection{Arthritic Mice (CIA)}

Extensive pathological changes were observed in the animals in this group. The most obvious alterations included the following: heavy mononuclear infiltration in the tissues adjacent to the articulation and in the articulation itself; cartilage alterations, including cell infiltration, cartilage hyperplasia and pannus; and bone alterations in the subchondral bone and the epiphysis, including cell infiltration, erosion and inter-articular blending. The prominent and diffuse mononuclear infiltration is the likely cause of these changes. Fibroblast activity, collagen overproduction and small vessel angiogenesis were also commonly found in the arthritic animals (Figure 5).

\subsection{Alpha Asarone}

Most of the pathological changes observed in the arthritic mice were also present in the asarone-treated arthritic mice $(\mathrm{CIA}+\mathrm{A})$. However, approximately onethird of the animals in the asarone-treated group had significant polymorphonuclear infiltration at regions adjacent to the injured articulation that was lacking in the 

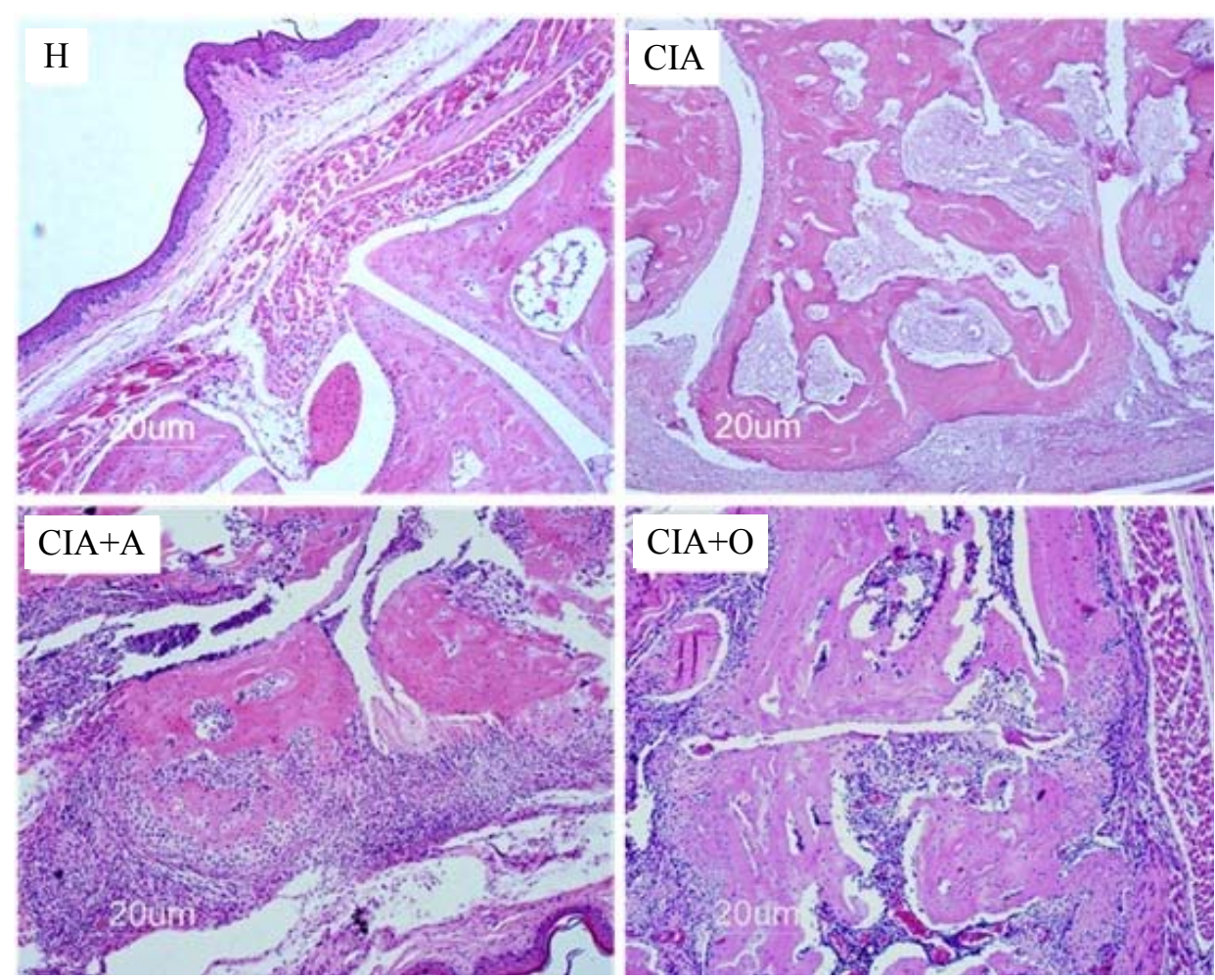

Figure 5. Histological appearance of the tarsal articulations of healthy DBA/1 mice, CIA mice (CIA), CIA mice treated with alpha asarone (CIA + A), and CIA mice treated with olive oil (CIA + O). Articular alterations, including cell proliferation and infiltration, cartilage destruction, and bone erosion and fusion, are clearly evident in the CIA groups. Haematoxylin-eosin, 10×.

control arthritic mice (Figure 5), suggesting the active recruitment of these cells into the inflamed articulation (see Section 4).

\subsection{Olive Oil Mice}

Arthritic mice treated with olive oil $(\mathrm{CIA}+\mathrm{O})$ showed changes similar to those observed in the asarone-treated arthritic mice (Figure 5), including some polymorphonuclear infiltration close to the articular structures.

\subsection{Figure 6 Illustrates the Most Prominent Changes in the Rear Footpads of CIA Mice}

These changes included heavy infiltration by mononuclear cells around and within the articulation that occurred simultaneously with bone and cartilage destruction, osteoclast hyperplasia, pannus formation, profuse angiogenesis, and fibroblast hyperactivity with the overproduction of reticular and collagen fibres. These changes are characteristic of both injury and tissue repair.

\subsection{Dexamethasone-Treated Mice}

Compared with the articulation of the untreated CIA mice, the articulations of the CIA mice treated with dexamethasone (CIA + D) showed distinct histological improvement, including much less cell infiltration in and around the articulation and cartilage and bone regeneration. Nonetheless, some inter-articular fusion, synovial inflammation and cartilage hyperplasia remained after 48 days of treatment (Figure 7).

\subsection{Neutrophils}

Healthy mice showed a significant number of neutrophils only within the bone marrow. CIA mice exhibited clusters of neutrophils adjacent to the injured articulation. Similarly, asarone-treated CIA mice had high numbers of neutrophils in and around the inflamed articulation. The CIA mice treated with olive oil (CIA + O) had a phenotype similar to that of the CIA mice (Figure 8).

\subsection{Anti-Collagen Antibodies}

Although neither BALB/c nor C57BL/6 mice developed CIA within the period examined (167 days), they produced high levels of anti-collagen antibodies by the end of this period.

As expected, DBA/1 mice also produced anti-collagen antibodies, and the levels were proportional to the time of immunisation. At the end of the experiment (on day 67 post-immunisation), CIA mice showed higher antibody 

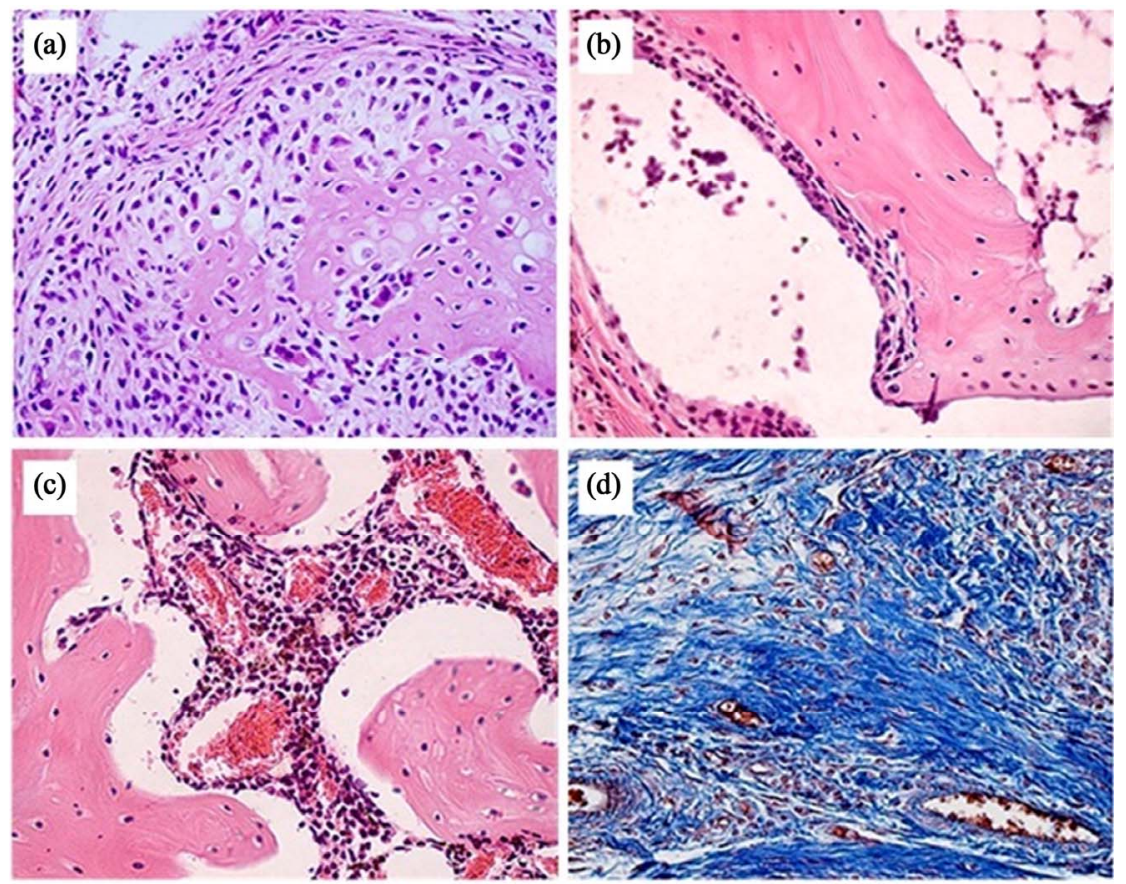

Figure 6. Four pathological characteristics of CIA in the mice that are similar to those observed in RA included the following: (a) Extended mononuclear infiltration within the subchondral bone starting at the cartilage articulation, combined with bone erosion and osteoclast hyperplasia; (b) Pannus on the articular structures; (c) Profuse angiogenesis; and (d) Fibroblast hyperactivity with overproduction of reticular and collagen fibres, which are a sign of tissue restoration.

levels than those detected in the healthy group (OD $2.268 \pm 0.218$ vs. $0.032 \pm 0.012, \mathrm{P}=0.001)$. Interestingly, CIA mice treated with alpha asarone produced lower levels of antibodies than the arthritic mice (OD $1.056 \pm$ 0.197 vs. OD $2.268 \pm 0.218, \mathrm{P}=0.001)$; however, this effect was also observed in the arthritic mice treated with olive oil alone, the vehicle for delivery of alpha asarone (OD $1.105 \pm 0.280$ ). Dexamethasone treatment led to the lowest anti-CII levels observed in the study. All of these results are shown in Figure 9.

\section{DISCUSSION}

Rheumatoid arthritis is a chronic degenerative disease that affects articulations. Clinical symptoms include stiffness of the hand and foot articulations, articular inflammation, bone erosion, pain, widespread vasculitis, including coronary artery disease, severe lung fibrosis, fatigue, and depression [31-33].

Despite extensive studies on rheumatoid arthritis (RA), the aetiology of this disease is still not completely understood. It is accepted that environmental factors (smoking, for instance), genetic polymorphisms, and conditions, such as advanced age and gender (women more than men), contribute to its development; thus, the origin of RA is multifactorial $[34,35]$. In humans, the inflamed articulations show variable numbers of lymphocytes, macrophages, neutrophils, and fibroblasts in the articular cartilage, with overproduction of collagen and proteoglycans. The presence of antibodies to collagen and proteoglycan and of rheumatoid factor in the synovia of affected articulations indicates that complement and neutrophils may participate in the damage [36,37].

Articular damage in RA begins with the activation and proliferation of synovial macrophages and fibroblasts immediately following an autoimmune or infectious event. This creates an inflammatory environment that promotes lymphocyte infiltration and endothelial cell proliferation. With time, the inflamed synovial tissue expands to produce an invasive tissue called pannus that destroys cartilage and bone. At the same time, a series of cytokines, chemokines, proteases, growth factors and angiogenic factors are released by the infiltrating cells, thereby enhancing the articular destruction [23,38-41].

To gain insight into the immunopathology of RA, several animal models have been developed. Of these models, collagen-induced arthritis (CIA) in mice is the most widely used because it exhibits many pathological features of RA. CIA is elicited in genetically susceptible strains of mice by immunisation with type II collagen (CII) emulsified in complete Freund's adjuvant (CIICFA). In the present study, neither C57BL/6 (I-A ${ }^{b}$ ) nor 

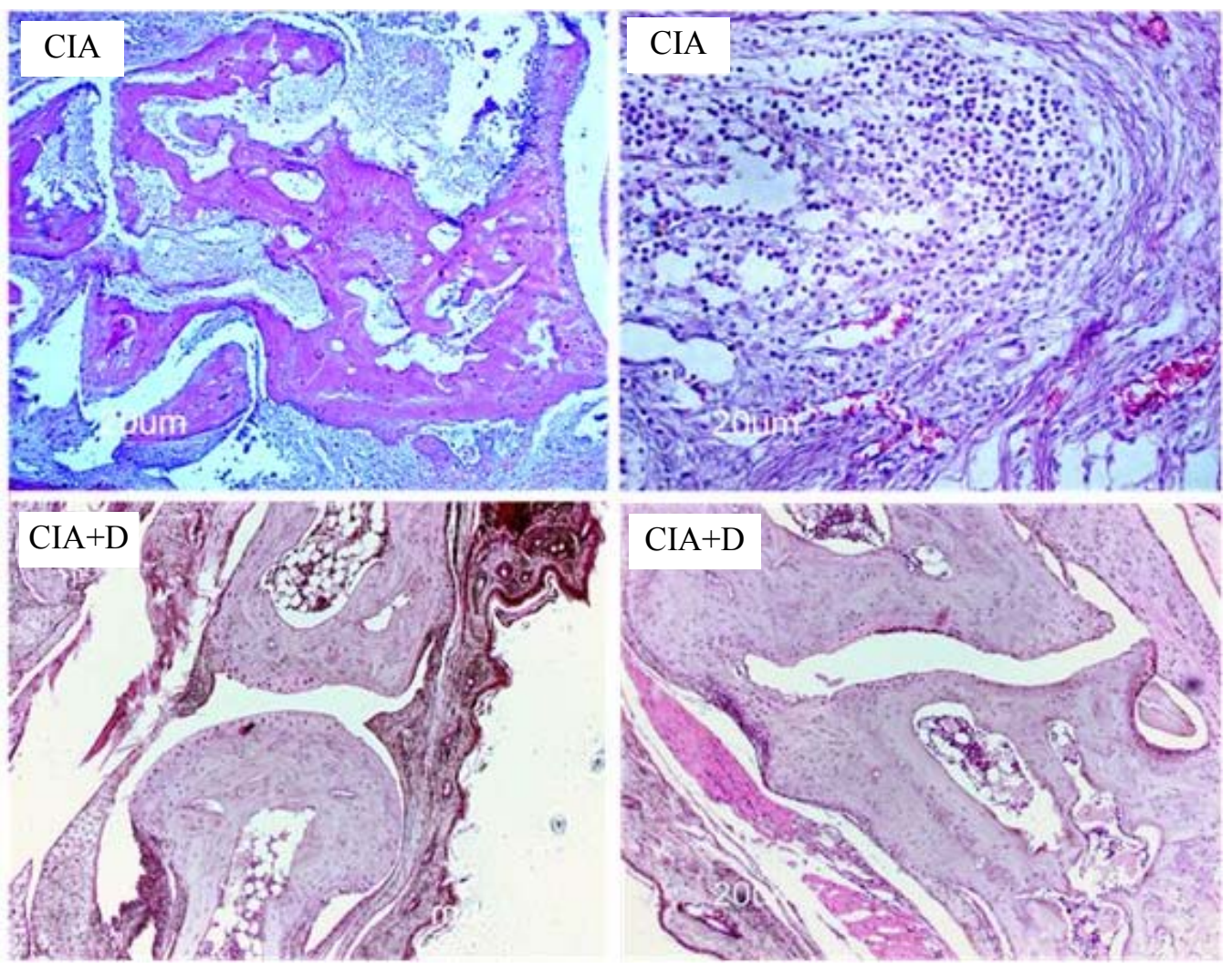

Figure 7. Effect of dexamethasone on CIA. The two upper photographs were taken of a mouse with CIA. The image on the left shows the most prominent CIA-induced changes in the hind footpad, including heavy cell infiltration in the connective tissue around and within the articulation, bone erosion, hyperplasia and destruction of the articular cartilage and the synovial layer. The image on the right depicts the mononuclear nature of the cell infiltrate, the reticular (collagen) response of tissue fibroblasts, and the angiogenic activity in the injured articular tissue. The two lower photographs show the histology of two articulations from a CIA mouse treated with dexamethasone $(\mathrm{CIA}+\mathrm{D})$. Compared to the untreated CIA articulation, the $\mathrm{CIA}+\mathrm{D}$ articulations show obvious histological improvement, including a much lower degree of cell infiltration in and around the articulation and cartilage and bone regeneration. However, some inter-articular fusion, synovial inflammation and cartilage hyperplasia continued to be observed at this time of the study (48 days of treatment).
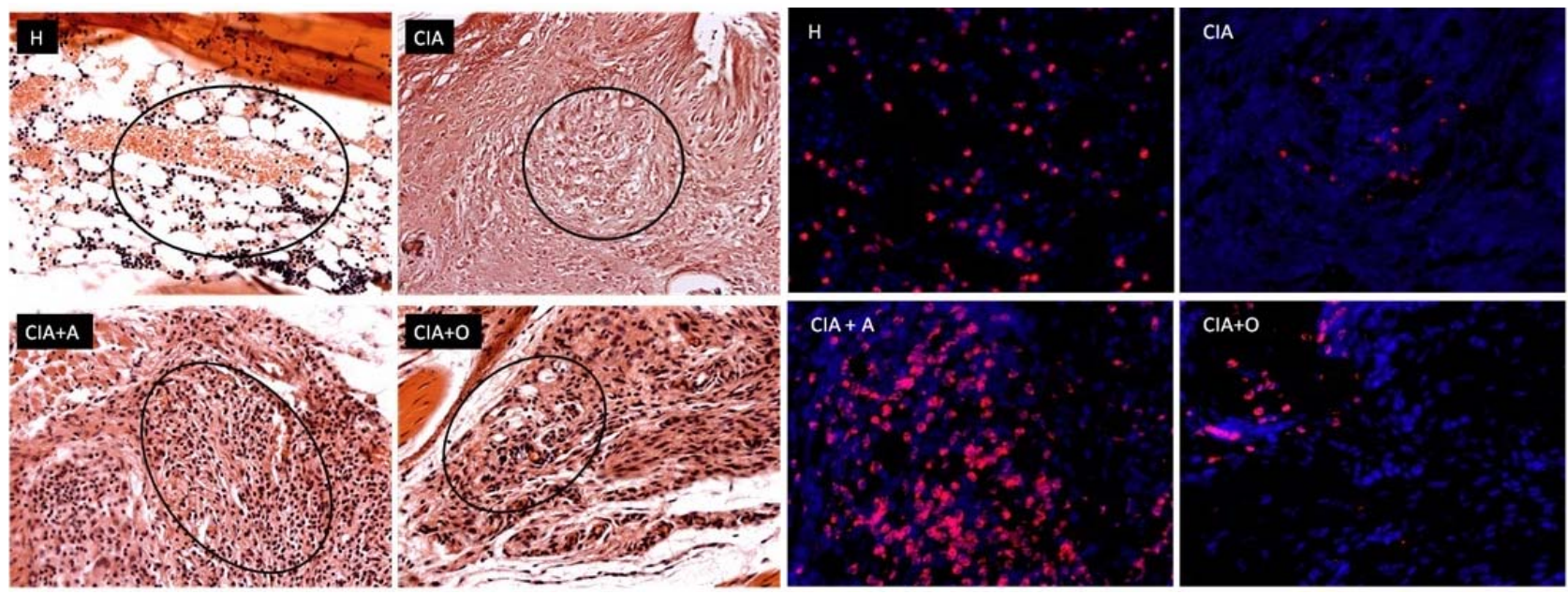

Figure 8. Representative rear footpad sections of healthy $(\mathrm{H})$, CIA, asarone-treated CIA (CIA+A) and olive oil-treated CIA (CIA+O) mice stained with haematoxylin-eosin (left 4-image panel), and contiguous tissue sections stained for neutrophils $(1 / 4+\mathrm{GR}-1+)($ red fluorescence, 20×). Circles on the upper sections indicate the approximate location of the neutrophils shown in the right 4-image panel. 


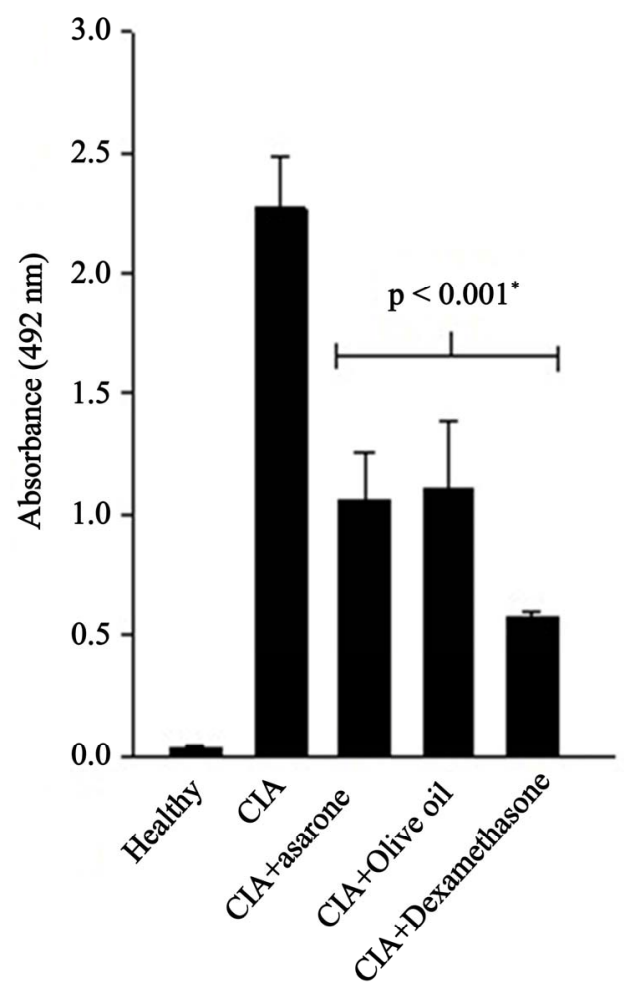

Figure 9. Anti-CII antibody levels in the sera of healthy DBA/1, CIA, and CIA mice left untreated or treated with $\alpha$-asarone, olive oil or dexamethasone for 48 days (67 days after primary immunisation with CII). Immunisation with CII induced a high anti-CII humoural response. Compared with the CIA group, all treatments led to a significant decrease in CII antibody levels $(\mathrm{P}<0.001)$.

BALB/c (I-A $\left.{ }^{\mathrm{d}}\right)$ mice developed CIA during the time of the study (up to 167 days), even though they developed high levels of anti-CII antibodies within this period. By contrast, DBA-1 (I-A $\left.{ }^{\mathrm{q}}\right)$ mice developed CIA with a frequency higher than $80 \%$; these data confirm that $\mathrm{MHC}$ genes are involved in the immune response to CII [42-43]. Lymphoproliferation of CIA DBA-1 mice when challenged in vitro with CII confirmed the participation of cell-mediated immunity (CMI) in the initiation of CIA. The high degree of activation of splenocytes in CIA DBA-1 mice in the absence of Con-A or antigen indicated a high basal level of activation that was most likely due to the strong inflammatory environment induced by CII-CFA.

In our study, we attempted to ameliorate the symptoms of CIA in the mice using alpha asarone, a prospective anti-inflammatory drug endowed with proven hypocholesterolaemic and cholelitholytic properties $[8,9]$.

We found that under the conditions used in this study, alpha asarone did not have any anti-inflammatory effects on CIA in the mice. The idea that alpha asarone might have anti-inflammatory effects came from studies on statins, drugs that, in addition to their hypocholesterolaemic effects, also have anti-inflammatory effects in rodent models of arthritis [44,45]. Both alpha asarone and statins are inhibitors of the 3-hydroxy-3-methylglutaryl coenzyme A reductase (HMG-CoA reductase) [10], which appears to be the basis for their hypocholesterolaemic and cholelitholytic effects [8,9]. It is clear that further investigation that involves testing different dosages, different lengths of treatment, and different administration routes is needed before a conclusive assessment of the effectiveness of alpha asarone can be made.

The high number of neutrophils in the CIA lesions of some of the mice treated with alpha asarone may be a meaningful finding. This neutrophil accumulation might be due to the pro-inflammatory environment in the arthritic lesions where chemokines are abundant, or the neutrophils might be recruited in response to the presence of antigen-antibody-complement complexes in the lesions $[36,37]$. Both possibilities deserve further investtigation. Th17 cells have been found in the inflamed articulations and blood of patients with rheumatoid arthritis. IL-17 has multiple effects on many cells, including neutrophils, macrophages, fibroblasts, epithelial cells, endothelial cells and mesenchymal cells, deregulating transcription factors that are involved in the synthesis of pro-inflammatory cytokines (IL-1, IL-6, GMCSF, TNF), chemokines, and osteogenic factor (responsible for bone resorption). The combined effect of these molecules leads to inflammation and chronic destruction of the articulation [46-48]. However, although neutrophils may cause tissue damage after granule exocytosis, they may also participate in the process of tissue remodelling, and this latter possibility perhaps explains their presence in the CIA lesions of mice treated with alpha asarone. In this case, alpha asarone might participate in neutrophil recruitment, which could lead to beneficial effects that would require more than 67 days (the length of our study) to manifest themselves.

The anti-inflammatory activity of dexamethasone in CIA observed in the present study is a known property of this drug. For this reason, dexamethasone has been used in the treatment of clinical and experimental RA [18,20, 49,50]. The drug appears to have pleiotropic effects that range from membrane stability to the inhibition of synthesis of inflammatory mediators, including cytokines (IL-1, TNF, IL-6, IL-8) [51], prostanoids [52], and signalling proteins linked to pro-inflammatory transcripttion factors [53]. Dexamethasone can also down regulate growth factors, including vascular endothelial growth factor and fibroblast growth factor [54]. In the current study, treatment with dexamethasone resulted in adequate anti-inflammatory control. 
Another interesting finding was the decrease in antiCII antibody levels in the animals that were treated with alpha asarone or olive oil; as asarone was dissolved in olive oil, this could have been an effect of the vehicle. Olive oil treatment also resulted in a decrease in the pad thickness of mice with CIA, although this decrease was not statistically significant $(\mathrm{P}>0.05)$. Only a couple of reports on the anti-inflammatory activity of olive oil in rodent arthritis have been published [14]. The beneficial effects of olive oil have been related to the antioxidant and anti-inflammatory effects of the oleic acids and polyphenols found in the oil. Specific polyphenols have been implicated in altering the levels of prostanoids (thromboxane A2 and PGE), the activity of cyclooxygenase 2 (Cox 2) [55], and the synthesis of PGE2 and vascular endothelial growth factor (VEGF) [56]. These activities may help control inflammation, atherogenesis, neoplasia, hyperlypidaemia, and angiogenesis.

The present study confirms the utility of the CIA mouse model for testing the effectiveness (or ineffectiveness) of prospective drugs for the treatment of RA.

\section{ACKNOWLEDGEMENTS}

This study received financial support from Secretaría de Investigación y Posgrado (SIP) of Instituto Politécnico Nacional, México. Authors are fellow-holders of SNI (LRP, ORE), IPN (LRP, ORE and PAP), and CONACYT (MAV). EBV is on leave of absence from Instituto Nacional de Psiquiatría "Dr. Ramón de la Fuente Muñiz", México. Authors thank Drs. Elba Reyes-Maldonado, Sergio Islas Trujillo, and Javier Rangel-Moreno, for their help in different parts of the study.

\section{REFERENCES}

[1] Martínez, M. (1992) Las plantas medicinales de México. 6th Edición, Editorial Botas, México.

[2] Sánchez-Reséndiz, J., Lerdo de Tejada, A., González-Vite, J. et al. (1980) Acción hipocolesterolemiante de Guatteria gaumeri. Traditional Medicine, 3, 20-22.

[3] Enríquez, R.G., Chávez, M.A. and Jáuregui, F. (1980) Propenylbenzenes from Guatteria gaumeri. Phytochemistry, 19, 2024-2025. doi:10.1016/0031-9422(80)83029-9

[4] Mandoki, J.J., Krumm-Heller, C., Vega-Noverola, J., et al. (1980) Isolation of alpha-asarone from the bark of Guatteria gaumeri (Elemuy) and the study of its hypocholesterolemic effect. IV National Congress of Pharmacology, Merida, 18-20 March 1980.

[5] Seshadri, T.R. and Thiruvengadam, T.R. (1950) A new synthesis of asarone. Proceedings of the Indian Academy of Science, 32A, 110-113.

[6] Díaz, F., Contreras, I., Flores, R., et al. (1990) An efficient synthesis of alpha asarone. Organic Preparations and Procedures International, 23, 133-138. doi:10.1080/00304949109458299

[7] Poplawski, J., Lozowicka, B., Dubis, A.T., et al. (2000)
Synthesis and hypolipidemic and antiplatelet activities of alpha-asarone isomers in humans (in vitro), mice (in vivo), and rats (in vivo). Journal of Medicinal Chemistry, 43, 3671-3676. doi:10.1021/jm000905n

[8] Gómez, C., Chamorro, G., Chávez, M.A., et al. (1987) Effect de l'alpha-asarone sur hypercholesterolemic et la cholelitiasis experimentales. Planta Medica Phytotherapy, 21, 279-284.

[9] Garduño, L., Salazar, M., Salazar, S., et al. (1997) Hypolipidaemic activity of alpha-asarone in mice. Journal of Ethnopharmacology, 55, 161-163. doi:10.1016/S0378-8741(96)01492-4

[10] Rodríguez-Páez, L., Juárez-Sánchez, M., Antúnez-Solís, J., et al. (2003) $\alpha$-asarone inhibits HMG-CoA reductase, lowers serum LDL-cholesterol levels and reduces biliary CSI in hypercholesterolemic rats. Phytomedicine, 10, 397-402. doi:10.1078/0944-7113-00274

[11] Estruch, R. (2010) Anti-inflammatory effects of the Mediterranean diet: The experience of the PREDIMED study. Proceedings of the Nutrition Society, 69, 333-340. doi: $10.1017 / \mathrm{S} 0029665110001539$

[12] Waterman, E. and Lockwood, B. (2007) Active components and clinical applications of olive oil. Alternative Medicine Review, 12, 331-342.

[13] Papageorgiou, N., Tousoulis, D., Psaltopoulou, T., et al. (2011) Divergent anti-inflammatory effects of different oil acute consumption on healthy individuals. European Journal of Clinical Nutrition, 65, 514-519.

[14] Impellizzeri, D., Esposito, E., Mazzon, E., et al. (2011) Oleuropein aglycone, an olive oil compound, ameliorates development of arthritis caused by injection of collagen type II in mice. Journal of Pharmacology and Experimental Therapeutics, 339, 859-869.

[15] Jaffré, B., Watrin, A., Loeuille, D., et al. (2003) Effects of antiinflamatory drugs on arthritic cartilage: A high-frequency quantitative ultrasound study in rats. Arthritis \& Rheumatism, 48, 1594-1601. doi:10.1002/art.11023

[16] Yang, Y.H., Morand, E.F., Getting, S.J., et al. (2004) Modulation of inflammation and response dexamethasone by annexin 1 in antigen-induced arthritis. Arthritis \& Rheumatism, 50, 976-984. doi:10.1002/art.20201

[17] Islander, U., Jochems, C., Stubelius, A., et al. (2011) Combined treatment with dexamethasone and raloxifene totally abrogates osteoporosis and joint destruction in experimental postmenopausal arthritis. Arthritis Research \& Therapy, 13, R96. doi:10.1186/ar3371

[18] Anderson, R., Franch, A., Castell, M., et al. (2010) Liposomal encapsulation enhances and prolongs the anti-inflammatory effects of water-soluble dexamethasone phosphate in experimental adjuvant arthritis. Arthritis Research \& Therapy, 12, R147. doi:10.1186/ar3089

[19] Kroot, E.J., Huisman, A.M., Van Zeben, J., et al. (2006) Oral pulsed dexamethasone therapy in early rheumatoid arthritis: A pilot study. Annals of the New York Academy of Sciences, 1069, 300-306. doi:10.1196/annals.1351.028

[20] Bos, W.H., Dijkmans, B.A., Boers, M., et al. (2010) Effect of dexamethasone on autoantibody levels and arthritis development in patients with arthralgia: A radomised 
trial. Annals of the Rheumatic Diseases, 69, 571-574. doi:10.1136/ard.2008.105767

[21] Brand, D.D. (2005) Rodent models of rheumatoid arthritis. Comparative Medicine, 55, 114-122.

[22] Brand, D.D., Latham, K.A. and Rosloniec, E.F. (2007) Collagen-induced arthritis. Nature Protocols, 2, 12691275. doi:10.1038/nprot.2007.173

[23] Andersson, A.K., Li, C. and Brennan, F.M. (2008) Recent developments in the immunobiology of rheumatoid arthritis. Arthritis Research \& Therapy, 10, 24. doi:10.1186/ar2370

[24] Asquith, D.L., Miller, A.M., McIcnnes, J.B. et al. (2009) Animal models of rheumatoid arthritis. European Journal of Immunology, 39, 1991-2058. doi:10.1002/eji.200939578

[25] Nguyen, T.G., Little, C.B., Yenson, V.M., et al. (2010) Anti-IgD antibody attenuates collagen-induced arthritis by selectively depleting mature B-cells and promoting immune tolerance. Journal of Autoimmunity, 35, 86-97. doi:10.1016/j.jaut.2010.03.003

[26] Shaw, J., Chen, B., Wooley, P., et al. (2011) Anti-inflammatory and anti-arthritic effects of a novel leflunomide analogue, UTL-5b (GBL-5b). American Journal of Biomedical Sciences, 3, 31-39. doi:10.5099/aj110100031

[27] Inglis, J.J., Šimelyte, E., McCann, F.E., et al. (2008) Protocol for the induction of arthritis in C57BL/6 mice. $\mathrm{Na}$ ture Protocols, 4, 612-618. doi:10.1038/nprot.2008.19

[28] Laemmli, U.K. (1970) Cleavage of structural proteins during the assembly of the head of bacteriophage T4. Nature, 227, 680-685. doi:10.1038/227680a0

[29] Seraffini-Fracassini, A. and Smith, J.W. (1974) Collagen. The structure and biochemistry of cartilage. Churchill Livingstone, Edinburgh.

[30] Sheehan, D.C. and Hrapchak, B.B. (1980) Theory and practice of histotechnology. 2nd Edition, The CV Mosby Company, St Louis.

[31] Vinaccia, S., Tobón, S., Moreno, S.-P., et al. (2005) Evaluación de la calidad de vida en pacientes con diagnóstico de artritis reumatoide. International Journal of Psychology and Psychological Therapy, 5, 45-59.

[32] Aletaha, D., Neogi, T., Silman, A.J., et al. (2010) Rheumatoid arthritis classification criteria: An American College of Rheumatology/European League against Rheumatism collaborative initiative. Arthritis \& Rheumatism, 62, 2569-2581. doi:10.1002/art.27584

[33] Hewlett, S., Sanderson, T., May, J., et al. (2011) "I'm hurting, I want to kill myself": Rheumatoid arthritis flare is more than a high joint count-An international patient perspective on flare where medical help is sought. Rheumatology, Oxford.

[34] Díaz-Jouanen, E., Abud-Mendoza, C., Garza-Elizondo, M.A., et al. Recommendations for the medical treatment of rheumatoid arthritis. Revista de Investigación Clínica, 57, 735-755.

[35] Pratt, A.G., Issacs, J. and Mattey, D.L. (2009) Current concepts in the pathogenesis of early rheumatoid arthritis. Best Practice \& Research Clinical Rheumatology, 23, 3738. doi:10.1016/j.berh.2008.08.002
[36] Low, J.M. and Moore, T.L. (2005) A role for the complement system in rheumatoid arthritis. Current Pharmaceutical Design, 11, 655-670. doi:10.2174/1381612053381936

[37] Okroj, M., Heinegård, D., Holmdahl, R., et al. (2007) Rheumatoid arthritis and the complement system. Annals of Medicine, 39, 517-530. doi: $10.1080 / 07853890701477546$

[38] Firestein, G.S. (2005) Pathogenesis of rheumatoid arthritis: How early is early? Arthritis Research \& Therapy, 7, 157-159. doi:10.1186/ar1780

[39] Rindfleisch, J.A. and Muller, D. (2005) Diagnosis and management of rheumatoid arthritis. American Family Physician, 72, 1037-1047.

[40] Otero, M. and Goldring, M.B. (2007) Cells of the synovium in rheumatoid arthritis. Chondrocytes. Arthritis Research \& Therapy, 9, 220. doi:10.1186/ar2292

[41] Rosloniec, E.F., Brand, D.D., Myers, L.K., et al. (1997) An HLA-DR1 transgene confers susceptibility to collagen-induced arthritis elicited with human type II collagen. Journal of Experimental Medicine, 185, 1113-1122. doi:10.1084/jem.185.6.1113

[42] Rosloniec, E.F., Brand, D.D., Myers, L.K., et al. (1998) Induction of autoimmune arthritis in HLA-DR4 (DRB1* 0401) transgenic mice by immunization with human and bovine type II collagen. Journal of Immunology, 160, 2573-2578.

[43] Kamradt, T. and Frey, O. (2010) Arthritis: Where are the T cells? Arthritis Research \& Therapy, 12, 122. doi:10.1186/ar3008

[44] Barsante, M.M., Roffê, E., Yokoro, C.M., et al. (2005) Anti-inflammatory and analgesic effects of atorvastatin in a rat model of adjuvant-induced arthritis. European Journal of Pharmacology, 516, 282-289. doi:10.1016/j.ejphar.2005.05.005

[45] Yamagata, T., Kinoshita, K., Nozaki, Y., et al. (2010) Effects of pravastatin in murine collagen-induced arthritis. British Journal of Clinical Pharmacologyi, 69, 85-94.

[46] Lubberts, E., Koenders, M.I. and Van den Berg, W.B. (2005) The role of T cell interleukin-17 in conducting destructive arthritis. Lessons from animal models. Arthritis Research \& Therapy, 7, 29-37. doi:10.1186/ar1550

[47] Raza, K., Falciani, F., Curnow, S.J., et al. (2005) Early rheumatoid arthritis is characterized by a distinct and transient synovial fluid cytokine profile of T cell and stromal cell origin. Arthritis Research \& Therapy, 7, R784R795. doi:10.1186/ar1733

[48] Lundy, S.K., Sarkar, S., Tesmer, L.A., et al. (2007) Cells of the synovium in rheumatoid arthritis. T lymphocytes. Arthritis Research \& Therapy, 9, 202. doi:10.1186/ar2107

[49] Earp, J.C., Dubois, D.C., Molano, D.S., et al. (2008). Modeling corticosteroid effects in a rat model of rheumatoid arthritis: Mechanistic pharmacodynamic model for dexamethasone effects in Lewis rats with collageninduced arthritis. Journal of Pharmacology and Experimental Therapeuticsi, 326, 546-554. doi:10.1124/jpet.108.137372

[50] Liu, X.M., Quan, L.D., Tian, J., et al. (2008) Synthesis 
and evaluation of a well-defined HPMA copolymerdexamethasone conjugate for effective treatment of rheumatoid arthritis. Pharmaceutical Research, 25, 29102919. doi:10.1007/s11095-008-9683-3

[51] De, A., Blotta, H.M., Mamoni, R.L., et al. (2002) Effects of dexamethasone on lymphocyte proliferation and cytokine production in rheumatoid arthritis. Journal of Rheumatology, 29, 46-51.

[52] Santini, G., Patrignani, P., Sciulli, M.G., et al. (2001) The human pharmacology of monocyte cyclooxygenase 2 inhibition by cortisol and synthetic glucocorticoids. Pharmacology \& Therapeutics, 70, 475-483. doi:10.1067/mcp.2001.119213

[53] Toh, M.L., Yang, Y., Leech, M., et al. (2004) Expression of mitogen-activated protein kinase phosphatase 1, a negative regulator of the mitogen protein kinases, in rheumatoid arthritis; up-regulation by interleukin-1beta and glucocorticoids. Arthritis \& Rheumatism, 50, 3118-
3128. doi:10.1002/art.20580

[54] Harding, G., Mak, Y.T., Evans, B., et al. (2006) The effects of dexamethasone and dehydroepiandrosterone (DHEA) on cytokines and receptor expression in a human osteoblastic cell line: Potential steroid-sparing role for DHEA. Cytokine, 36, 57-68. doi:10.1016/j.cyto.2006.10.012

[55] Trefiletti, G., Togna, A.R, Latina, V., et al. (2011) 1-Phenyl-6,7-dihydroxy-isochroman suppresses lipopolysaccharide-induced pro-inflammatory mediator production in human monocytes. British Journal of Nutrition, 27, $1-4$.

[56] Terzuoli, E., Donnini, S., Giachetti, A., et al. (2010) Inhibition of hypoxia inducible factor-1alpha by dihydroxyphenylethanol, a product from olive oil, blocks microsomal prostaglandin-E synthase-1/vascular endothelial growth factor expression and reduces tumor angiogenesis. Clinical Cancer Research, 16, 4207-4216. doi:10.1158/1078-0432.CCR-10-0156 